\title{
Directional selection on cold tolerance does not constrain plastic capacity in a butterfly
}

\author{
Kristin Franke, Anneke Dierks and Klaus Fischer*
}

\begin{abstract}
Background: Organisms may respond to environmental change by means of genetic adaptation, phenotypic plasticity or both, which may result in genotype-environment interactions $(G \times E)$ if genotypes differ in their phenotypic response. We here specifically target the latter source of variation (i.e. $\mathrm{G} \times \mathrm{E}$ ) by comparing plastic responses among lines of the tropical butterfly Bicyclus anynana that had been selected for increased cold tolerance and according controls. Our main aim here was to test the hypothesis that directional selection on cold tolerance will interfere with plastic capacities.

Results: Plastic responses to temperature and feeding treatments were strong, with e.g. higher compared to lower temperatures reducing cold tolerance, longevity, pupal mass, and development time. We report a number of statistically significant genotype-environment interactions (i.e. interactions between selection regime and environmental variables), but most of these were not consistent across treatment groups. We found some evidence though for larger plastic responses to different rearing temperatures in the selection compared to the control lines, while plastic responses to different adult temperatures and feeding treatments were overall very similar across selection regimes.
\end{abstract}

Conclusion: Our results indicate that plastic capacities are not always constrained by directional selection (on cold tolerance) and therefore genetic changes in trait means, but may operate independently.

Keywords: Artificial selection, Bicyclus anynana, Constraint, Genetic adaptation, Genotype by environment interaction, Phenotypic plasticity, Temperature stress resistance

\section{Background}

Temperature is considered one of the most important selective agents, and consequently research on temperature stress resistance has attracted much interest over recent decades [1-5]. Temperature-stress resistance refers to an organism's ability to cope with stressfully high or low temperatures, and is considered a key factor for explaining the distribution and abundance of species [6,7]. Enhanced resistance to temperature stress can be reached by means of phenotypic plasticity, i.e. nongenetic physiological changes as a direct response to environmental variation, or genetic adaptation $[5,8]$. Genetic variation in temperature stress resistance has been commonly reported in natural systems, with e.g. tropical species showing a higher heat but a lower cold tolerance than temperate-zone species and vice versa

\footnotetext{
* Correspondence: klaus.fischer@uni-greifswald.de

Department of Animal Ecology, Zoological Institute and Museum, University of Greifswald, J.-S. Bachstraße 11/12, D-17489, Greifswald, Germany
}

[1,3,4,6,7,9-13]. Likewise, high-altitude populations typically show a lower heat but a higher cold tolerance than low-altitude populations [14]. Such geographic variation in fitness-related traits provides strong evidence that these patterns have been shaped by natural selection $[14,15]$. Several species are also known to respond readily to artificial selection on thermal tolerance traits, providing direct experimental proof for genetic adaptation in temperature stress resistance (e.g. [2,16,17]).

In addition to genetic adaptation, phenotypic plasticity provides a further efficient mechanism to cope with temperature variation [18]. We here define phenotypic plasticity as the set of different phenotypes that may be produced by a single genotype in direct response to different environmental conditions [8]. Plastic responses can be induced during development (developmental plasticity) or in the adult stage (adult acclimation; $[5,19,20]$ ), and beneficial effects of a brief exposure to less extreme temperatures are referred to as rapid hardening $[1,21,22]$. Studies 
on plastic responses in temperature stress resistance revealed that cooler rearing or adult temperatures increase cold but decrease heat resistance and vice versa, comprising a near universal pattern of putatively adaptive phenotypic plasticity $[1,6,23]$. For instance, groups of a tropical butterfly reared in a common environment, but being exposed for two days to either $20^{\circ} \mathrm{C}$ or $27^{\circ} \mathrm{C}$ in the adult stage, differed subsequently by a factor $2-3$ in heat tolerance [23]. Thus, plastic changes in thermal tolerance may be induced within short periods of time, and are highly effective means to deal with temperature variation.

Both genetic variation and phenotypic plasticity may finally interact with one another, resulting in genotypeenvironment interactions ( $\mathrm{G} \times \mathrm{E}$ ). We here use $\mathrm{G} \times \mathrm{E}$ to refer to different degrees by which individual genotypes are able to respond to environmental variation [24]. Consequently, a significant $\mathrm{G} \times \mathrm{E}$ demonstrates that some genotypes are more plastic than others [14]. Quantifying such variation is consequently used to explore genetic variation in phenotypic plasticity [25-28]. G x E has been reported for e.g. insect growth and development, fecundity, and immune function [29,30], and is considered to contribute substantially to the maintenance of the genetic variation of traits [31,32]. Although interactions between genotypes and environmental conditions may thus represent a significant source of variation, our respective knowledge is still fairly limited.

Against this background we here investigate $\mathrm{G} \times \mathrm{E}$ in control and selection lines of the tropical nymphalid butterfly Bicyclus anynana (Butler, 1879). This species is known to respond readily to temperature manipulations by phenotypic plasticity in temperature stress resistance as well as in an array of other traits [20,23]. Different genotypes of $B$. anynana had been previously produced by applying artificial selection to chill-coma recovery time, yielding highly divergent lines [17]. Chill-coma recovery time is the time an individual needs to regain mobility after cold exposure, and is considered a reliable proxy of climatic cold adaptation [6,23,32,33]. This trait is furthermore known to respond readily to selection as well as to ambient temperatures [2,16,23,34]. We thus exploit the genetic variation generated through artificial selection to investigate whether genotypes substantially varying in cold tolerance respond differentially to environmental manipulations. This explicit focus on $\mathrm{G} \times \mathrm{E}$ contrasts with earlier studies from our group investigating responses and correlated responses to selection [34], effects of selection in the adult stage throughout the life cycle [35], environmental effects on cold tolerance [19], or effects of inbreeding [17]. Specifically we here test the hypotheses that lines selected for increased cold tolerance show reduced plastic responses in cold tolerance (and possibly further traits) compared to control lines. This expectation rests on the assumption that the cold- tolerant lines should benefit less from strong plastic responses in case of critically low temperatures compared to control lines. Further, trait values may already be close to their physiologically determined maximum, constraining further plastic increases.

In order to induce plastic responses we exposed selection and control lines to different rearing and acclimation temperatures as well as to different feeding regimes, and measured the respective effects on cold tolerance and additionally on development and longevity. Some of the concomitant effects reported here are hardly novel, though including environmental manipulations is evidently necessary to investigate $G \times$ E. Furthermore this enables us to test a number of additional hypotheses, namely that higher temperatures are predicted to speed up development and to reduce pupal mass, longevity, and cold tolerance $[5,20,36,37]$. A period of starvation is, based on earlier results, predicted to reduce longevity but to leave cold tolerance unaffected ([23,36,37]; see Table 1 for specific hypotheses).

As the initial design of the selection experiment included inbreeding as a factor, we furthermore consider effects of inbreeding in our study. However, based on the fact that the lines were allowed to mate randomly after the different inbreeding levels had been established, no effects of inbreeding are expected anymore (see further below). Selection regime is, based on previous results, also expected to yield no effects on traits other than cold tolerance [34]. We finally include sex as a factor in our analyses, predicting that females show a slower larval but a faster pupal development, a higher pupal mass, and a higher longevity compared to males ([23], Table 1).

\section{Methods}

\section{Study organism}

Bicyclus anynana (Butler 1897; Nymphalidae, Satyrinae) is a tropical fruit-feeding butterfly, ranging from southern Africa to Ethiopia [38]. The species inhabits regions with alternating dry and wet seasons, and shows accordingly two seasonal morphs. During the colder dry season (ca. $18^{\circ} \mathrm{C}$, May until November) the species has rather uniform wing patterns and small eyespots, while it exhibits large eyespots and bright bands on both wings in the warmer wet season (ca. $23^{\circ} \mathrm{C}$, December until April; [39]). During the dry season reproduction ceases and butterflies do not mate before the onset of the next wet season [29]. Females are relatively monandrous, though multiple mating occurs in the field and in the laboratory [40]. A stock population of B. anynana was founded at Greifswald University in 2007 from several hundred individuals derived from a well-established stock population at Leiden University, The Netherlands. The latter was founded in 1988 from over 80 gravid females collected at a single locality in Nkhata Bay, Malawi. To maintain high levels of 
Table 1 Hypotheses for the main effects of several factors on larval time, pupal time, larval growth rate, pupal mass, chill-coma recovery time, and longevity

\begin{tabular}{|c|c|c|c|c|c|c|}
\hline \multirow[t]{2}{*}{ Traits } & \multicolumn{6}{|c|}{ Factors } \\
\hline & Selection regime & Inbreeding & Rearing temperature & Sex & Adult temperature & Feeding regime \\
\hline Larval time & No & No & $20^{\circ} \mathrm{C}>27^{\circ} \mathrm{C}$ & Females > Males & - & - \\
\hline Pupal time & No & No & $20^{\circ} \mathrm{C}>27^{\circ} \mathrm{C}$ & Males > Females & - & - \\
\hline Growth rate & No & No & $27^{\circ} \mathrm{C}>20^{\circ} \mathrm{C}$ & Males $>$ Females & - & - \\
\hline Pupal mass & No & No & $20^{\circ} \mathrm{C}>27^{\circ} \mathrm{C}$ & Females > Males & - & - \\
\hline $\begin{array}{l}\text { Chill-coma recovery } \\
\text { time }\end{array}$ & Controls > Selected Lines & No & $27^{\circ} \mathrm{C}>20^{\circ} \mathrm{C}$ & No & $27^{\circ} \mathrm{C}>20^{\circ} \mathrm{C}$ & No \\
\hline Longevity & No & No & $20^{\circ} \mathrm{C}>27^{\circ} \mathrm{C}$ & Females > Males & $20^{\circ} \mathrm{C}>27^{\circ} \mathrm{C}$ & Control > Starvation \\
\hline
\end{tabular}

heterozygosity several hundred adults are reared in each generation [41]. For this experiment animals from the Greifswald stock population were used.

\section{Experimental design}

We here used 12 selection lines that had been previously established at the Department of Animal Ecology, Greifswald University [34,35]. Selection lines for increased cold stress resistance (shorter chill-coma recovery times) and according unselected controls had been derived from three different levels of inbreeding, using a full-sib breeding design: outbred controls $(\mathrm{C})$ having resulted from matings between unrelated butterflies, inbreeding 1 (I1) having resulted from matings between full sibs, and inbreeding 2 (I2) having resulted from matings between full sibs in two consecutive generations. We used ca. 120 full-sib families each for inbreeding levels C, I1, and I2 [34]. At the start of the selection experiment though butterflies were pooled across families within inbreeding levels. Thus, during the course of the selection experiment (10 generations) mating was random within lines. Per inbreeding level, four lines were set up, two for increased cold stress resistance and two unselected controls. This design resulted in a total of 6 selection and 6 control lines (for details see $[17,34]$ ). Lines had been kept without selection under standard rearing conditions for 4 generations prior to this experiment. Several hundred butterflies were reared per line in each generation. For the current experiment eggs were collected from all 12 lines. Eggs were thereafter randomly divided among a low $\left(20^{\circ} \mathrm{C}\right)$ and a high $\left(27^{\circ} \mathrm{C}\right)$ rearing temperature $(70 \pm 5 \%$ relative humidity and photoperiod of L12:D12 throughout). The two temperatures chosen are similar to the ones this species experiences during the wet and dry season in the field, respectively [29]. Larvae were reared in sleeve-like gauze cages, using 10 replicate cages per line and rearing temperature (resulting in a total of 240 cages), and a standard density of $20\left(20^{\circ} \mathrm{C}\right)$ and $40\left(27^{\circ} \mathrm{C}\right)$ larvae per cage, respectively. The higher density per cage at $27^{\circ} \mathrm{C}$ was due to the need for more individuals at this temperature (see below). Care was taken though that developing larvae were never exposed to any food shortage, being fed on young maize plants ad libitum throughout. Resulting pupae were collected daily, weighed one day after pupation, and were then individually transferred to small plastic cups (volume $125 \mathrm{ml}$ ). For all animals we scored larval time (from egg-laying until pupation, thus including egg development), pupal time (from pupation until adult eclosion), larval growth rate (ln pupal mass/larval time), and pupal mass (measured on day 2 after pupation).

Following adult eclosion, all butterflies were marked individually and afterwards once again randomly divided among $20^{\circ} \mathrm{C}$ and $27^{\circ} \mathrm{C}$, resulting in four rearing by adult temperature groups per selection line $\left(20-20^{\circ} \mathrm{C}, 20-27^{\circ} \mathrm{C}\right.$, $27-20^{\circ} \mathrm{C}, 27-27^{\circ} \mathrm{C}$ ). While the butterflies reared at $27^{\circ} \mathrm{C}$ were a last time divided among two feeding treatments, being fed with banana (control) or water only (starvation), all animals reared at $20^{\circ} \mathrm{C}$ were fed with banana ad libitum (to keep the size of the experiment manageable). On day two after eclosion, we tested 24-52 individuals per sex, treatment group and line for chill-coma recovery time, resulting in a total number of 4772 butterflies. Therefore, all butterflies were transferred individually to plastic cups $(125 \mathrm{ml})$, arranged on a tray in a randomized block design, and then exposed to $1^{\circ} \mathrm{C}$ for $19 \mathrm{~h}$ to induce a chill coma. Recovery time (the time until the butterflies were able to stand up) was scored in a climate cell at $20^{\circ} \mathrm{C}$. Observations were terminated after 60 minutes. Butterflies that had not recovered by then were given the maximal recovery time of 60 minutes. This applied to $<5 \%$ of the butterflies tested.

Thus, we used the same technique for measuring chillcoma recovery time as during selection [35]. This trait is considered a reliable proxy of climatic cold adaptation, is largely independent of the method used to induce a chill coma, and has been used successfully in B. anynana 
before [24]. To afterwards score longevity the butterflies reared at $20^{\circ} \mathrm{C}$ were returned to their respective adult temperatures and fed ad libitum, while the butterflies reared at $27^{\circ} \mathrm{C}$ were all transferred to $27^{\circ} \mathrm{C}$ (due to space limitations) and fed as outlined above until death.

\section{Statistical analyses}

Data on larval time, pupal time, pupal mass, growth rate, chill coma recovery time, and longevity were analyzed with mixed model analyses of variance (ANOVAs), with selection regime (selection vs. control), level of inbreeding $(\mathrm{C}, \mathrm{I} 1, \mathrm{I})$, rearing temperature $\left(20\right.$ vs. $\left.27^{\circ} \mathrm{C}\right)$, adult temperature $\left(20\right.$ vs. $\left.27^{\circ} \mathrm{C}\right)$, adult feeding regime (control vs. starvation, for animals reared at $27^{\circ} \mathrm{C}$ only), and/or sex as fixed factors. Replicate line was nested within selection regime and inbreeding level, and replicate cage was nested within replicate line, inbreeding level and selection regime. Both latter factors were included as random effects. Minimum adequate models were constructed by sequentially removing non-significant interaction terms. Due to differences in the experimental set-up across rearing temperatures (see above), three different analyses were run for chill-coma recovery time and longevity. For chill-coma recovery time we analyzed all individuals together (note that effects of adult feeding regime were non-significant) and separated by rearing temperature, as different adult feeding regimes were only applied to animals reared at $27^{\circ} \mathrm{C}$. For longevity, effects of rearing temperature were analyzed in animals fed ad libitum and kept as adults at $27^{\circ} \mathrm{C}$, effects of adult temperature were analyzed in animals reared at $20^{\circ} \mathrm{C}$ (as only in these different adult temperatures were used throughout), and effects of adult feeding regime were analyzed in animals reared at $27^{\circ} \mathrm{C}$ (as only in these different adult feeding regimes were applied). Significant differences between inbreeding levels were located using Tukey's HSD posthoc test. When presenting the statistical results we start with the main effects and continue with interactions, which can be problematic in case of strong interactive effects. Therefore, main effects are interpreted cautiously in case of significant interactions. All statistical tests were performed by using JMP (4.0.0) or Statistica (6.1). Throughout, all means are given \pm 1 SE.

\section{Results}

\section{Larval time}

Larval time was significantly affected by rearing temperature, sex, replicate line and replicate cage, but not by selection regime and inbreeding level (Table 2A). Larval time was much longer at the lower compared to the higher rearing temperature $(50.6 \pm 0.06 \mathrm{~d}>27.4 \pm 0.05 \mathrm{~d}$; Figure $1 \mathrm{~A})$, and was longer in females than in males $(40.2 \pm 0.06 \mathrm{~d}>$ $37.8 \pm 0.05 \mathrm{~d}$ ). The absolute difference in larval time between males and females was smaller at $27^{\circ} \mathrm{C}(26.4 \pm 0.06$ d vs. $28.3 \pm 0.07$ d) than at $20^{\circ} \mathrm{C}(49.2 \pm 0.09$ d vs. $52.0 \pm$
$0.09 \mathrm{~d}$ ), as indicated by the significant rearing temperature by sex interaction. The sexual difference was particularly small in the unselected controls reared at $27^{\circ} \mathrm{C}$ (significant selection regime * rearing temperature * sex interaction; Figure 1B). The significant interaction between selection regime and rearing temperature reveals a significantly longer larval time in control than in selection lines at $20^{\circ} \mathrm{C}$ $(51.0 \pm 0.09 \mathrm{~d}>50.2 \pm 0.09 \mathrm{~d})$, which was not the case at $27^{\circ} \mathrm{C}(27.5 \pm 0.06 \mathrm{~d}=27.3 \pm 0.07 \mathrm{~d}$; Tukey HSD $)$. At the higher rearing temperature, larval time was longer in the control compared to the inbred groups (C: $28.0 \pm 0.08 \mathrm{~d}$ > I1: $27.1 \pm 0.08 \mathrm{~d}=\mathrm{I} 2: 27.1 \pm 0.08 \mathrm{~d}$ ), while there were no significant differences at $20^{\circ} \mathrm{C}$ (C: $50.5 \pm 0.1 \mathrm{~d}=\mathrm{I} 1: 50.3 \pm$ $0.1 \mathrm{~d}=\mathrm{I} 2: 50.9 \pm 0.1 \mathrm{~d}$; Tukey HSD; significant inbreeding level * rearing temperature interaction). Both above patterns though were not consistent across all groups (significant selection regime " inbreeding level " rearing temperature interaction; Figure 1A).

\section{Pupal time}

Pupal time was significantly influenced by rearing temperature, sex, replicate cage and replicate line, but not by selection regime and inbreeding level (Table 2B). Pupal time was longer at the lower compared to the higher rearing temperature $(11.9 \pm 0.02 \mathrm{~d}>7.0 \pm 0.01 \mathrm{~d})$, and was longer in males than in females $(9.7 \pm 0.02 \mathrm{~d}>9.2 \pm 0.02$ d). A significant rearing temperature by sex interaction indicates that sexual differences were slightly more pronounced at $27^{\circ} \mathrm{C}(7.4 \pm 0.02 \mathrm{~d}>6.7 \pm 0.02 \mathrm{~d})$ than at $20^{\circ} \mathrm{C}$ $(12.1 \pm 0.03 \mathrm{~d}>11.6 \pm 0.03 \mathrm{~d}$; Figure $1 \mathrm{C})$. At the higher rearing temperature, pupal time increased with increasing inbreeding level $(\mathrm{C}: 6.8 \pm 0.02 \mathrm{~d}<\mathrm{I} 1: 7.1 \pm 0.02 \mathrm{~d}<\mathrm{I} 2: 7.2$ $\pm 0.02 \mathrm{~d}$ ), while at the lower rearing temperature inbreeding level had no significant effect (C: $11.9 \pm 0.03 \mathrm{~d}=\mathrm{I} 1$ : $11.9 \pm 0.03 \mathrm{~d}=\mathrm{I} 2: 11.8 \pm 0.03 \mathrm{~d}$; Tukey HSD; significant inbreeding level * rearing temperature interaction). Furthermore, inbreeding effects on pupal time were more pronounced in males $(\mathrm{C}: 9.6 \pm 0.03 \mathrm{~d}<\mathrm{I} 1: 9.8 \pm 0.03 \mathrm{~d}=$ I2: $9.8 \pm 0.03 \mathrm{~d}$ ) than in females (C: $9.1 \pm 0.03 \mathrm{~d}=\mathrm{I} 1: 9.2$ $\pm 0.03 \mathrm{~d}=\mathrm{I} 2: 9.2 \pm 0.03 \mathrm{~d}$; Tukey HSD; significant inbreeding level * sex interaction). Consequently, inbreeding effects were largely restricted to males reared at $27^{\circ} \mathrm{C}$ (significant inbreeding level * rearing temperature * sex interaction; Figure 1C). The significant interaction between selection regime, inbreeding level and rearing temperature indicates that differences between selection and control lines were basically limited to the outbred control group when reared at $27^{\circ} \mathrm{C}$ (Figure 1D).

\section{Growth rate}

Growth rate was also significantly affected by rearing temperature, sex, replicate line and replicate cage, but not by selection regime and inbreeding level (Table 2C). Growth rate was higher at the higher compared to the 
Table 2 Results of nested ANOVAs for larval time (A), pupal time (B), larval growth rate (C) and pupal mass (D)

\begin{tabular}{|c|c|c|c|c|}
\hline A) Larval time & $D F$ & MS & $F$ & $P$ \\
\hline Selection Regime & 1,6 & 205.6 & 1.0 & 0.3453 \\
\hline Inbreeding Level & 2,6 & 87.2 & 0.4 & 0.6614 \\
\hline $\begin{array}{l}\text { Replicate Line [Sel. Reg. } \\
\text { \& Inbr.] }\end{array}$ & 6,110 & 216.0 & 9.4 & $<0.0001$ \\
\hline $\begin{array}{l}\text { Repl. Cage [Sel., Inbr. } \\
\text { \& Repl.] }\end{array}$ & 108,4640 & 23.5 & 3.8 & $<0.0001$ \\
\hline Rearing Temperature & 1,4640 & 565906.0 & 90265.2 & $<0.0001$ \\
\hline Sex & 1,4640 & 5780.5 & 922.0 & $<0.0001$ \\
\hline $\begin{array}{l}\text { Selection Reg. * Inbreeding } \\
\text { Level }\end{array}$ & 2,6 & 236.5 & 1.2 & 0.3635 \\
\hline Selection Reg. * Rearing Temp. & 1,4640 & 107.4 & 17.1 & $<0.0001$ \\
\hline Selection Reg. * Sex & 1,4640 & 11.2 & 1.8 & 0.1819 \\
\hline $\begin{array}{l}\text { Inbreeding Level * Rearing } \\
\text { Temp. }\end{array}$ & 2,4640 & 139.2 & 22.2 & $<0.0001$ \\
\hline Inbreeding Level * Sex & 2,4640 & 14.4 & 2.3 & 0.1007 \\
\hline Rearing Temp. * Sex & 1,4640 & 219.6 & 35.0 & $<0.0001$ \\
\hline $\begin{array}{l}\text { Sel. Reg. * Inbreed. * Rear. } \\
\text { Temp. }\end{array}$ & 2,4640 & 49.9 & 8.0 & 0.0004 \\
\hline $\begin{array}{l}\text { Sel. Reg. * Rearing Temp. } \\
\text { * Sex }\end{array}$ & 1,4640 & 25.0 & 25.0 & 0.0457 \\
\hline Error & 4640 & 6.3 & & \\
\hline B) Pupal time & $D F$ & MS & $F$ & $P$ \\
\hline Selection Regime & 1,6 & 1.4 & 0.1 & 0.7627 \\
\hline Inbreeding Level & 2,6 & 9.2 & 0.6 & 0.5567 \\
\hline $\begin{array}{l}\text { Replicate Line [Sel. Reg. } \\
\text { \& Inbr.] }\end{array}$ & 6,111 & 15.6 & 11.7 & $<0.0001$ \\
\hline $\begin{array}{l}\text { Repl. Cage [Sel., Inbr. } \\
\text { \& Repl.] }\end{array}$ & 108,4639 & 1.4 & 2.5 & $<0.0001$ \\
\hline Rearing Temperature & 1,4639 & 24740.3 & 45323.1 & $<0.0001$ \\
\hline Sex & 1,4639 & 376.4 & 689.6 & $<0.0001$ \\
\hline $\begin{array}{l}\text { Selection Reg. * Inbreeding } \\
\text { Level }\end{array}$ & 2,6 & 1.7 & 0.1 & 0.8888 \\
\hline $\begin{array}{l}\text { Selection Reg. * Rearing } \\
\text { Temp. }\end{array}$ & 1,4639 & 0.2 & 0.4 & 0.5196 \\
\hline Selection Reg. * Sex & 1,4639 & 0.8 & 8.8 & 0.3708 \\
\hline $\begin{array}{l}\text { Inbreeding Level * Rearing } \\
\text { Temp. }\end{array}$ & 2,4639 & 12.8 & 23.4 & $<0.0001$ \\
\hline Inbreeding Level * Sex & 2,4639 & 3.0 & 5.5 & 0.0041 \\
\hline Rearing Temp. * Sex & 1,4639 & 13.6 & 24.9 & $<0.0001$ \\
\hline $\begin{array}{l}\text { Sel. Reg. * Inbreed. * Rear. } \\
\text { Temp. }\end{array}$ & 2,3941 & 3.5 & 6.3 & 0.0018 \\
\hline $\begin{array}{l}\text { Inbreed. * Rear. Temp. } \\
\text { * Sex }\end{array}$ & 2,4639 & 2.2 & 4.1 & 0.0173 \\
\hline Error & 4639 & 0.5 & & \\
\hline C) Growth rate & $D F$ & MS & $F$ & $P$ \\
\hline Selection Regime & 1,6 & 5.7 & 0.1 & 0.7710 \\
\hline Inbreeding Level & 2,6 & 40.9 & 0.7 & 0.5514 \\
\hline
\end{tabular}

Table 2 Results of nested ANOVAs for larval time (A), pupal time (B), larval growth rate (C) and pupal mass (D) (Continued)

\begin{tabular}{|c|c|c|c|c|}
\hline $\begin{array}{l}\text { Replicate Line [Sel. Reg. } \\
\text { \& Inbr.] }\end{array}$ & 6,109 & 68.1 & 8.2 & $<0.0001$ \\
\hline $\begin{array}{l}\text { Repl. Cage [Sel., Inbr. } \\
\text { \& Repl.] }\end{array}$ & 108,4641 & 8.6 & 4.3 & $<0.0001$ \\
\hline Rearing Temperature & 1,4641 & 79417.9 & 40343.3 & $<0.0001$ \\
\hline Sex & 1,4641 & 154.2 & 78.3 & $<0.0001$ \\
\hline $\begin{array}{l}\text { Selection Reg. * Inbreeding } \\
\text { Level }\end{array}$ & 2,6 & 43.2 & 0.7 & 0.5353 \\
\hline $\begin{array}{l}\text { Selection Reg. * Rearing } \\
\text { Temp. }\end{array}$ & 1,4641 & 2.1 & 1.1 & 0.3012 \\
\hline Selection Reg. * Sex & 1,4641 & 0.4 & 0.2 & 0.6716 \\
\hline $\begin{array}{l}\text { Inbreeding Level * Rearing } \\
\text { Temp. }\end{array}$ & 2,4641 & 33.0 & 16.8 & $<0.0001$ \\
\hline Inbreeding Level * Sex & 2,4641 & 0.6 & 0.3 & 0.7479 \\
\hline Rearing Temp. * Sex & 1,4641 & 31.3 & 15.9 & $<0.0001$ \\
\hline $\begin{array}{l}\text { Sel. Reg. * Inbreed. * Rear. } \\
\text { Temp. }\end{array}$ & 2,4641 & 8.8 & 4.5 & 0.0115 \\
\hline Error & 4641 & 2.0 & & \\
\hline D) Pupal mass & $D F$ & MS & $F$ & $P$ \\
\hline Selection Regime & 1,6 & 2366.2 & 0.6 & 0.4880 \\
\hline Inbreeding Level & 2,6 & 38734.6 & 8.9 & 0.0156 \\
\hline $\begin{array}{l}\text { Replicate Line [Sel. Reg. } \\
\text { \& Inbr.] }\end{array}$ & 6,110 & 4746.8 & 3.2 & 0.0064 \\
\hline $\begin{array}{l}\text { Repl. Cage [Sel., Inbr. } \\
\text { \& Repl.] }\end{array}$ & 108,4641 & 1527.4 & 3.8 & $<0.0001$ \\
\hline Rearing Temperature & 1,4641 & 156622.0 & 389.2 & $<0.0001$ \\
\hline Sex & 1,4641 & 1661991.0 & 4143.6 & $<0.0001$ \\
\hline $\begin{array}{l}\text { Selection Reg. * Inbreeding } \\
\text { Level }\end{array}$ & 2,6 & 231.4 & 0.1 & 0.9487 \\
\hline $\begin{array}{l}\text { Selection Reg. * Rearing } \\
\text { Temp. }\end{array}$ & 1,4641 & 949.7 & 2.4 & 0.1239 \\
\hline Selection Reg. * Sex & 1,4641 & 251.4 & 0.6 & 0.4286 \\
\hline $\begin{array}{l}\text { Inbreeding Level * Rearing } \\
\text { Temp. }\end{array}$ & 2,4641 & 1285.1 & 3.2 & 0.0407 \\
\hline Inbreeding Level * Sex & 2,4641 & 1253.2 & 3.1 & 0.0441 \\
\hline Rearing Temp. * Sex & 1,4641 & 295.4 & 0.7 & 0.3908 \\
\hline $\begin{array}{l}\text { Sel. Reg. * Inbreed. * Rear. } \\
\text { Temp. }\end{array}$ & 2,4641 & 2182.8 & 5.4 & 0.0044 \\
\hline Error & 4641 & 400.5 & & \\
\hline
\end{tabular}

Results for the effects of selection regime (selection vs. control), inbreeding level $(C, I 1,12)$, replicate line, replicate cage, rearing temperature $\left(20\right.$ vs. $\left.27^{\circ} \mathrm{C}\right)$, and sex on larval time (A), pupal time (B), larval growth rate (C), and pupal mass (D) in Bicyclus anynana. Replicate line was nested within selection regime and inbreeding level, and replicate cage was nested within replicate line, inbreeding level and selection regime. Both latter factors were included as random effects, while all others were considered fixed effects. Minimum adequate models were constructed by sequentially removing non-significant interaction terms. Significant $P$-values are given in bold. 

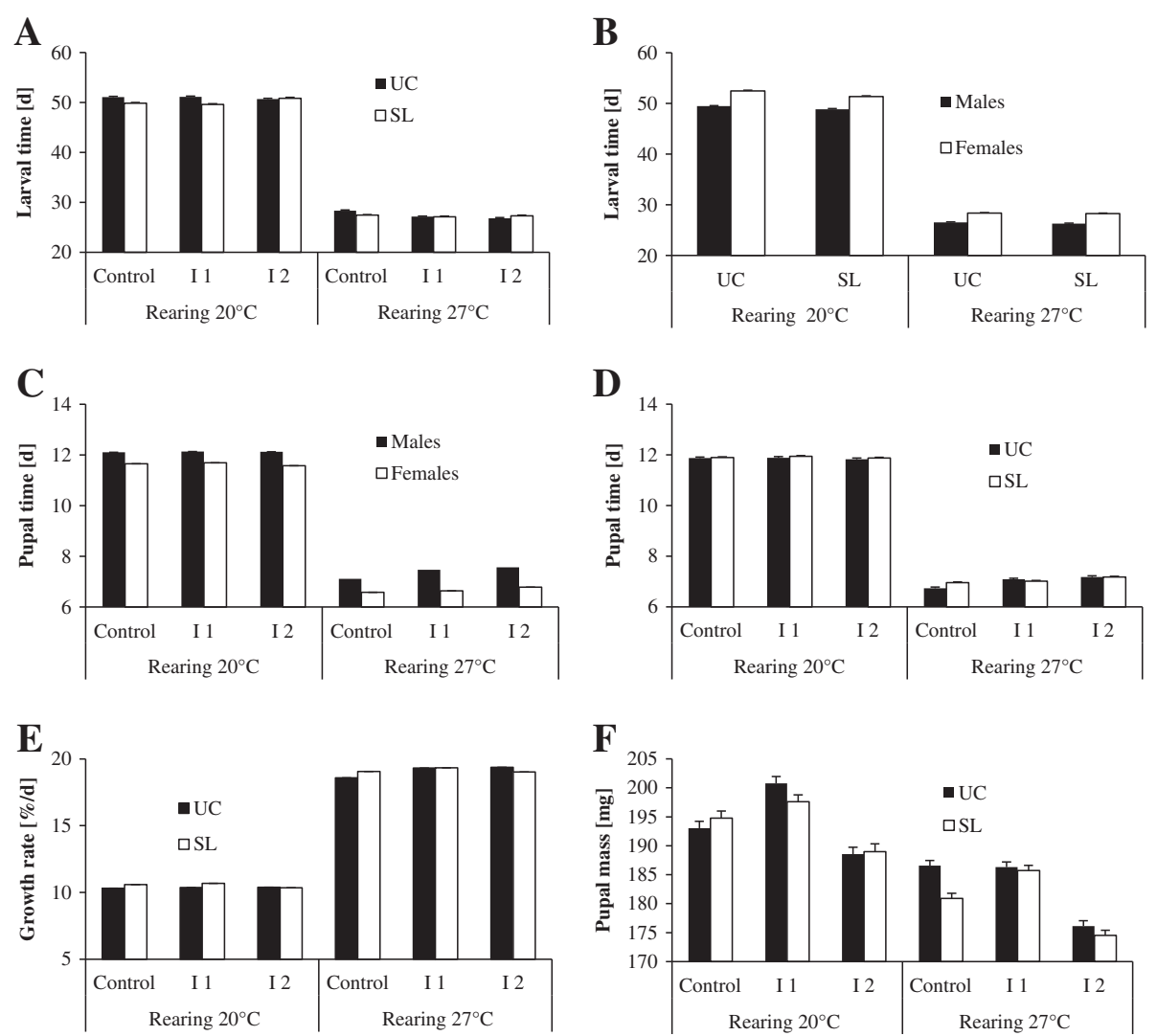

Figure 1 Variation in life-history traits (means $+1 \mathrm{SE}$ ) in relation to various factors in Bicyclus anynana. Larval time in relation to rearing temperature, inbreeding level and selection regime $(\mathbf{A})$, and in relation to rearing temperature, selection regime and sex (B); pupal time in relation to rearing temperature, inbreeding level and sex $(\mathbf{C})$, and in relation to rearing temperature, inbreeding level and selection regime (D); larval growth rate in relation to rearing temperature, inbreeding level and selection regime $\mathbf{( E )}$; and pupal mass in relation to rearing temperature, inbreeding level and selection regime (F). UC: unselected control; SL: selection line. Please note that in some cases standard errors are so small that error bars are not visible.

lower rearing temperature $(19.1 \pm 0.03 \% / \mathrm{d}>10.4 \pm$ $0.03 \% / \mathrm{d})$, and was higher in males than in females (14.9 \pm $0.03 \% / d>14.6 \pm 0.03 \% / d$ ). The significant interaction between rearing temperature and sex indicates a larger sexual difference in growth rates at $27^{\circ} \mathrm{C}$ (males: $19.4 \pm$ $0.04 \% / \mathrm{d}>$ females: $18.8 \pm 0.04 \% / \mathrm{d}$ ) than at $20^{\circ} \mathrm{C}$ (males: $10.5 \pm 0.05 \% / \mathrm{d}>$ females: $10.3 \pm 0.05 \% / \mathrm{d}$ ). Effects of inbreeding were found at the higher rearing temperature only (C: $18.8 \pm 0.04 \% / \mathrm{d}<\mathrm{I} 1: 19.3 \pm 0.04 \% / \mathrm{d}=\mathrm{I} 2: 19.2$ $\pm 0.05 \% / \mathrm{d})$, but not at the lower one (C: $10.4 \pm 0.06 \% / \mathrm{d}$ $=\mathrm{I} 1: 10.5 \pm 0.06 \% / \mathrm{d}=\mathrm{I} 2: 10.3 \pm 0.06 \% / \mathrm{d}$; Tukey HSD, significant inbreeding level * rearing temperature interaction). Moreover, clear evidence for effects of inbreeding on growth rates was restricted to the unselected control lines reared at $27^{\circ} \mathrm{C}$ (significant selection regime * inbreeding level * rearing temperature interaction; Figure 1E).

\section{Pupal mass}

Pupal mass differed significantly between inbreeding levels, rearing temperatures, sexes, replicate lines and replicate cages, but not in relation to selection regime (Table 2D). Pupal mass was highest in the inbreeding 1 group, followed by the outbred control and finally the inbreeding 2 group (I1: $192.7 \pm 1.72 \mathrm{mg}>$ C: $188.9 \pm$ $1.74 \mathrm{mg}>$ I2: $182.1 \pm 1.85 \mathrm{mg}$; Tukey HSD). The exact patterns though differed among the higher (I1: $186.0 \pm$ $0.63 \mathrm{mg}=\mathrm{C}: 183.9 \pm 0.64 \mathrm{mg}>\mathrm{I} 2: 175.4 \pm 0.65 \mathrm{mg})$ and the lower rearing temperature (I1: $199.3 \pm 0.83 \mathrm{mg}>\mathrm{C}$ : $193.9 \pm 0.85 \mathrm{mg}>\mathrm{I} 2: 188.7 \pm 0.91 \mathrm{mg}$; Tukey HSD; significant inbreeding level * rearing temperature interaction), and also among the sexes (males: I1: $173.1 \pm 0.73 \mathrm{mg}>\mathrm{C}$ : $168.0 \pm 0.73 \mathrm{mg}>\mathrm{I} 2: 163.0 \pm 0.77 \mathrm{mg}$; females: I1: $212.2 \pm$ $0.74 \mathrm{mg}=\mathrm{C}: 209.7 \pm 0.74 \mathrm{mg}>\mathrm{I} 2: 201.2 \pm 0.78 \mathrm{mg}$; significant inbreeding level * sex interaction). Effects of selection regime were absent except for the outbred controls reared at $27^{\circ} \mathrm{C}$, where pupal mass was higher in the unselected controls (significant selection regime * inbreeding level " rearing temperature interaction; Figure 1F). Pupal mass was overall higher at $20^{\circ} \mathrm{C}$ than at $27^{\circ} \mathrm{C}(194.0 \pm$ $0.50 \mathrm{mg}>181.8 \pm 0.37 \mathrm{mg}$ ), and was higher in females than in males $(207.7 \pm 0.44 \mathrm{mg}>168.0 \pm 0.43 \mathrm{mg})$. 
Table 3 Results of nested ANOVAs for chill-coma recovery time

\begin{tabular}{|c|c|c|c|c|}
\hline A) Chill-coma recovery time & $D F$ & MS & $F$ & $P$ \\
\hline Selection Regime & 1,6 & 24368.4 & 22.0 & 0.0032 \\
\hline Inbreeding Level & 2,6 & 1183.4 & 1.1 & 0.4005 \\
\hline Replicate Line [Sel. \& Inbr.] & 6,4738 & 1204.3 & 7.7 & $<0.0001$ \\
\hline Rearing Temperature & 1,4738 & 127708.0 & 815.0 & $<0.0001$ \\
\hline Adult Temperature & 1,4738 & 115643.0 & 738.0 & $<0.0001$ \\
\hline Sex & 1,4738 & $<0.1$ & $<0.1$ & 0.9926 \\
\hline Selection Reg. * Inbreeding Level & 2,6 & 192.6 & 0.2 & 0.8448 \\
\hline Selection Reg.* Rearing Temp. & 1,4738 & 480.4 & 3.1 & 0.0800 \\
\hline Selection Reg. * Adult Temp. & 1,4738 & 1327.2 & 8.5 & 0.0036 \\
\hline Selection Reg. * Sex & 1,4738 & 21.2 & 0.1 & 0.7131 \\
\hline Inbreeding Level * Rearing Temp. & 2,4738 & 797.1 & 5.1 & 0.0062 \\
\hline Inbreeding Level * Adult Temp. & 2,4738 & 692.4 & 4.4 & 0.0121 \\
\hline Inbreeding Level * Sex & 2,4738 & 254.0 & 1.6 & 0.1979 \\
\hline Rearing Temp. * Adult Temp. & 1,4738 & 1.2 & $<0.1$ & 0.9298 \\
\hline Rearing Temp. * Sex & 1,4738 & 1.8 & $<0.1$ & 0.9140 \\
\hline Adult Temp. * Sex & 1,4738 & 1384.2 & 8.8 & 0.0030 \\
\hline Sel. Reg. * Inbreed. * Rear. Temp. & 2,4738 & 910.95 & 5.8 & 0.0030 \\
\hline Sel. Reg. ${ }^{*}$ Inbreed. ${ }^{*}$ Adult Temp. & 2,4738 & 527.1 & 3.4 & 0.0347 \\
\hline Inbreed. * Rear. Temp. * Adult Temp. & 2,4738 & 504.9 & 3.2 & 0.0400 \\
\hline Rear. Temp. * Adult Temp. * Sex & 1,4738 & 1121.7 & 7.2 & 0.0075 \\
\hline Error & 4738 & 156.7 & & \\
\hline B) Animals reared at $20^{\circ} \mathrm{C}$ & $D F$ & MS & $F$ & $P$ \\
\hline Selection Regime & 1,6 & 6782.9 & 18.8 & 0.0049 \\
\hline Inbreeding Level & 2,6 & 213.8 & 0.6 & 0.5829 \\
\hline Replicate Line [Sel. \& Inbr.] & 6,1668 & 362.6 & 4.1 & 0.0004 \\
\hline Adult Temperature & 1,1668 & 44483.1 & 501.1 & $<0.0001$ \\
\hline Sex & 1,1668 & 1.3 & $<0.1$ & 0.9046 \\
\hline Selection Reg. ${ }^{*}$ Adult Temp. & 1,1668 & 1458.2 & 16.4 & $<0.0001$ \\
\hline Error & 1668 & 88.8 & & \\
\hline C) Animals reared at $27^{\circ} \mathrm{C}$ & $D F$ & MS & $F$ & $P$ \\
\hline Selection Regime & 1,6 & 23033.8 & 15.3 & 0.0079 \\
\hline Inbreeding Level & 2,6 & 2340.1 & 1.5 & 0.2859 \\
\hline Replicate Line [Sel. \& Inbreed.] & 6,3059 & 1506.7 & 7.9 & $<0.0001$ \\
\hline Adult Temperature & 1,3059 & 81814.6 & 427.7 & $<0.0001$ \\
\hline Adult Feeding Regime & 1,3059 & 5.6 & $<0.1$ & 0.8644 \\
\hline Sex & 1,3059 & 0.4 & $<0.1$ & 0.9620 \\
\hline Selection Reg. * Inbreeding Level & 2,6 & 959.2 & 0.6 & 0.5611 \\
\hline Selection Reg. * Adult Temp. & 1,3059 & 321.2 & 1.7 & 0.1952 \\
\hline Selection Reg. * Adult Feed. & 1,3059 & 66.0 & 0.3 & 0.5570 \\
\hline Selection Reg. * Sex & 1,3059 & 38.0 & 0.2 & 0.6557 \\
\hline Inbreeding Level * Adult Temp. & 2,305 & 1643.0 & 8.6 & 0.0002 \\
\hline Inbreeding Level * Adult Feed. & 2,3059 & 211.2 & 1.1 & 0.3317 \\
\hline Inbreeding Level * Sex & 2,3059 & 145.2 & 0.8 & 0.4682 \\
\hline
\end{tabular}

Table 3 Results of nested ANOVAs for chill-coma recovery time (Continued)

\begin{tabular}{lrrrc}
\hline Adult Temp. * Adult Feed. & 1,3059 & 231.8 & 1.2 & 0.2711 \\
Adult Temp. * Sex & 1,3059 & 3459.7 & 18.1 & $<\mathbf{0 . 0 0 0 1}$ \\
Adult Feed. * Sex & 1,3059 & 174.4 & 0.9 & 0.3397 \\
Sel. Reg. * Inbreed. * Adult Temp. & 2,3059 & 961.3 & 5.0 & $\mathbf{0 . 0 0 6 6}$ \\
Inbreed. * Adult Feed. * Sex & 2,3059 & 1243.4 & 6.5 & $\mathbf{0 . 0 0 1 5}$ \\
Adult Temp. * Adult Feed. * Sex & 1,3059 & 1362.7 & 7.1 & $\mathbf{0 . 0 0 7 6}$ \\
Error & 3059 & 191.3 & & \\
\hline
\end{tabular}

Results for the effects of selection regime (selection vs. control), inbreeding level $(C, 11,12)$, replicate line, rearing temperature $\left(20\right.$ vs. $\left.27^{\circ} \mathrm{C}\right)$, adult temperature $\left(20\right.$ vs. $\left.27^{\circ} \mathrm{C}\right)$, and sex on chill-coma recovery time in Bicyclus anynana (A). In Tables B (animals reared at $20^{\circ} \mathrm{C}$ ) and $\mathrm{C}$ (animals reared at $27^{\circ} \mathrm{C}$ ) data were analyzed separately for each rearing temperature, as different feeding treatments were employed in animals reared at $27^{\circ} \mathrm{C}$ only.

Consequently, the factor rearing temperature was dropped from both analyses, and the factor adult feeding regime (control vs. starvation) was added to Table C. For details see Material and Methods. Replicate line (random factor) was nested within selection regime and inbreeding level throughout. Minimum adequate models were constructed by sequentially removing non-significant interaction terms. Significant $P$-values are given in bold.

\section{Chill-coma recovery time}

Chill-coma recovery time was significantly affected by selection regime, rearing temperature, adult temperature, and replicate line, but not by inbreeding level and sex (Table 3A). Animals from the selection lines recovered faster than those from the control lines $(18.2 \pm 0.73 \mathrm{~min}>23.0 \pm 0.70 \mathrm{~min})$. Furthermore, animals reared at $20^{\circ} \mathrm{C}$ recovered faster than those reared at $27^{\circ} \mathrm{C}(15.2 \pm 0.31 \mathrm{~min}<26.0 \pm 0.23 \mathrm{~min})$, as was the case for animals acclimated to $20^{\circ} \mathrm{C}$ compared to $27^{\circ} \mathrm{C}$ $(15.4 \pm 0.27 \mathrm{~min}<25.8 \pm 0.27 \mathrm{~min})$. The significant interaction between selection regime and adult temperature reveals that the difference between selection lines and unselected controls was larger at $27^{\circ} \mathrm{C}(22.9 \pm$ $0.38 \mathrm{~min}$ versus $28.7 \pm 0.37 \mathrm{~min})$ than at $20^{\circ} \mathrm{C}(13.6 \pm$ $0.38 \mathrm{~min}$ versus $17.3 \pm 0.37 \mathrm{~min})$. Evidence for effects of inbreeding on chill-coma recovery time were restricted to animals reared at $27^{\circ} \mathrm{C}$ (C: $25.5 \pm 0.39 \mathrm{~min}=\mathrm{I} 2: 24.8$ $\pm 0.39 \mathrm{~min}<\mathrm{I} 1: 27.7 \pm 0.39 \mathrm{~min})$, while no effects of inbreeding were found in animals reared at $20^{\circ} \mathrm{C}$ (C: 14.4 $\pm 0.52 \mathrm{~min}=\mathrm{I} 2: 15.6 \pm 0.55 \mathrm{~min}=\mathrm{I} 1: 15.5 \pm 0.52 \mathrm{~min}$, Tukey HSD; significant inbreeding level * rearing temperature interaction). More specifically, the selection lines reared at $27^{\circ} \mathrm{C}$ caused the above pattern, while the unselected control lines showed neither at $20^{\circ} \mathrm{C}$ nor at $27^{\circ} \mathrm{C}$ a significant response to inbreeding, and while the selection lines reared at $20^{\circ} \mathrm{C}$ showed an increase in chill-coma recovery time with increasing inbreeding level (significant selection regime * inbreeding level * rearing temperature interaction; Figure 2A).

Likewise, inbreeding effects were only detectable in animals acclimated to $27^{\circ} \mathrm{C}(\mathrm{C}: 24.8 \pm 0.46 \mathrm{~min}=\mathrm{I} 2$ : $24.9 \pm 0.48 \mathrm{~min}<\mathrm{I} 1: 27.6 \pm 0.46 \mathrm{~min})$, but not in those acclimated to $20^{\circ} \mathrm{C}(\mathrm{C}: 15.1 \pm 0.46 \mathrm{~min}=\mathrm{I} 2: 15.5 \pm$ 

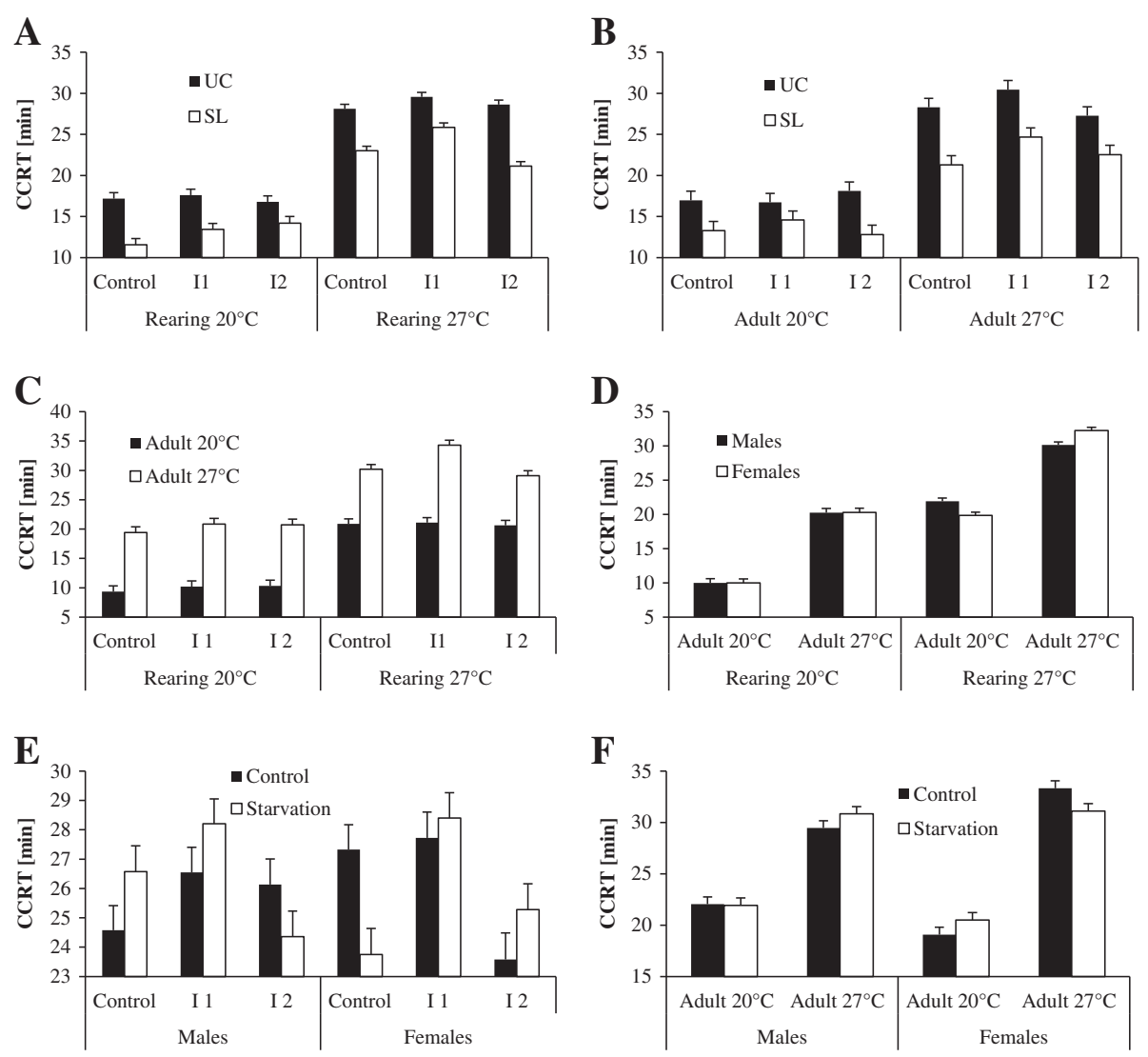

Figure 2 Variation in chill-coma recovery time (means $+1 \mathrm{SE}$ ) in Bicyclus anynana. Chill-coma recovery time in relation to rearing temperature, inbreeding level and selection regime (A), adult temperature, inbreeding level and selection regime (B), rearing temperature, inbreeding level and adult temperature $(\mathbf{C})$, rearing temperature, adult temperature and sex (D), sex, inbreeding level and adult feeding regime (E), and sex, adult temperature and adult feeding regime $(\mathbf{F})$. Graphs A-D refer to all animals, while graphs E-F refer only to the animals reared at $27^{\circ} \mathrm{C}$. UC: unselected control; SL: selection line; CCRT: Chill-coma-recovery-time.

$0.48 \min =\mathrm{I} 1: 15.7 \pm 0.46 \mathrm{~min}$; Tukey HSD; significant inbreeding level * adult temperature interaction). The lack of a comparable response in the butterflies acclimated to $20^{\circ} \mathrm{C}$ is caused by the unselected controls, showing an increase in recovery time with increasing inbreeding level (significant selection regime * inbreeding level " adult temperature interaction; Figure 2B). Overall, inbreeding effects were thus only detectable in the animals reared at and acclimated to $27^{\circ} \mathrm{C}$, but not in any other rearing by adult temperature group (significant inbreeding level " rearing temperature * adult temperature interaction; Figure 2C). The significant adult temperature by sex interaction indicates that males tended to show longer recovery times than females at the adult temperature of $20^{\circ} \mathrm{C}(16.0 \pm 0.38 \mathrm{~min}$ vs. 14.9 $\pm 0.38 \mathrm{~min})$, but shorter ones than females at $27^{\circ} \mathrm{C}(25.2$ $\pm 0.37 \mathrm{~min}$ vs. $26.3 \pm 0.39 \mathrm{~min})$. This pattern was restricted to animals reared at $27^{\circ} \mathrm{C}$ though, while sexual differences were absent in animals reared at $20^{\circ} \mathrm{C}$ (significant rearing temperature * adult temperature * sex interaction; Figure 2D).
When analyzing the animals reared at $20^{\circ} \mathrm{C}$ separately, selection regime, adult temperature and replicate line significantly affected chill-coma recovery time, but not inbreeding level and sex (Table 3B). Butterflies from the selection lines recovered quicker compared to unselected control butterflies $(13.2 \pm 0.70 \mathrm{~min}<17.2 \pm$ $0.66 \mathrm{~min}$ ), and adults kept at $20^{\circ} \mathrm{C}$ recovered much faster than those kept at $27^{\circ} \mathrm{C}(10.1 \pm 0.32 \mathrm{~min}<20.3 \pm$ $0.33 \mathrm{~min}$ ). All patterns are thus in full agreement with the above analysis. The interaction between selection regime and adult temperature indicates that differences in chill-coma recovery time between selection and control lines were more pronounced at the higher (17.4 \pm $0.47 \mathrm{~min}$ vs. $23.3 \pm 0.45 \mathrm{~min})$ compared to the lower adult temperature $(9.0 \pm 0.47 \mathrm{~min}$ vs. $11.1 \pm 0.45 \mathrm{~min})$.

In the animals reared at $27^{\circ} \mathrm{C}$, chill-coma recovery time was significantly affected by selection regime, adult temperature, and replicate line, but not by adult feeding regime, sex and inbreeding level (Table 3C). Butterflies from the selection lines had shorter recovery times than those from the unselected control lines $(23.3 \pm 0.99 \mathrm{~min}$ 
Table 4 Results of nested ANOVAs for longevity

\begin{tabular}{|c|c|c|c|c|}
\hline A) Longevity & $D F$ & MS & $F$ & $P$ \\
\hline Selection Regime & 1,6 & 234.0 & 0.6 & 0.4616 \\
\hline Replicate Line [Sel. \& Inbr.] & 6,3744 & 404.9 & 2.8 & 0.0109 \\
\hline Inbreeding Level & 2,6 & 146.3 & 0.4 & 0.6967 \\
\hline Rearing Temperature & 1,3744 & 264262.0 & 1806.6 & $<0.0001$ \\
\hline Sex & 1,3744 & 20.7 & 0.14 & 0.7070 \\
\hline Selection Reg. * Inbreeding Level & 2,6 & 2020.7 & 5.3 & 0.0440 \\
\hline Selection Reg. * Rearing Temp. & 1,3744 & 93.4 & 0.6 & 0.4242 \\
\hline Selection Reg. * Sex & 1,3744 & 52.2 & 0.4 & 0.5505 \\
\hline Inbreeding Level * Rearing Temp. & 2,3744 & 440.1 & 3.0 & 0.0495 \\
\hline Inbreeding Level * Sex & 2,3744 & 153.7 & 1.0 & 0.3498 \\
\hline Rearing Temp. * Sex & 1,3744 & 5324.0 & 36.4 & $<0.0001$ \\
\hline Sel. Reg. * Inbreed. * Rear. Temp. & 2,3744 & 472.0 & 3.2 & 0.0398 \\
\hline Sel. Reg. * Inbreed. ${ }^{*}$ Sex & 2,3744 & 940.0 & 6.4 & 0.0016 \\
\hline Sel. Reg. * Rearing Temp. * Sex & 1,3744 & 1.5 & $<0.1$ & 0.9202 \\
\hline Inbreed. * Rear. Temp. * Sex & 2,3744 & 178.4 & 1.2 & 0.2954 \\
\hline Four-way Interaction & 2,3744 & 932.1 & 6.4 & 0.0017 \\
\hline Error & 3744 & 146.3 & & \\
\hline B) Animals reared at $20^{\circ} \mathrm{C}$ & $D F$ & MS & $F$ & $P$ \\
\hline Selection Regime & 1,6 & 287.5 & 0.3 & 0.6267 \\
\hline Inbreeding Level & 2,6 & 174.0 & 0.2 & 0.8570 \\
\hline Replicate Line [Sel. Reg. \& Inbr.] & 6,1312 & 1106.3 & 4.8 & $<0.0001$ \\
\hline Adult Temperature & 1,1312 & 34430.6 & 148.6 & $<0.0001$ \\
\hline Sex & 1,1312 & 2568.9 & 11.1 & 0.0009 \\
\hline Selection Reg. ${ }^{*}$ Inbreeding Level & 2.6 & 1712.1 & 1.6 & 0.2864 \\
\hline Selection Reg. ${ }^{*}$ Adult Temp. & 1,1312 & 1726.0 & 7.5 & 0.0064 \\
\hline Selection Reg. * Sex & 1,1312 & 7.3 & $<0.1$ & 0.8589 \\
\hline Inbreeding Level * Adult Temp. & 2,1312 & 3034.3 & 13.5 & $<0.0001$ \\
\hline Inbreeding Level * Sex & 2,1312 & 131.9 & 0.6 & 0.5659 \\
\hline Adult Temperature * Sex & 1,1312 & 2762.2 & 12.6 & 0.0004 \\
\hline Sel. Reg. * Inbreed. * Sex & 2,1312 & 1395.7 & 6.0 & 0.0025 \\
\hline Error & 1312 & 231.6 & & \\
\hline C) Animals reared at $27^{\circ} \mathrm{C}$ & $D F$ & MS & $F$ & $P$ \\
\hline Selection Regime & 1,6 & 38.0 & 0.1 & 0.7603 \\
\hline Inbreeding Level & 2,6 & 142.8 & 0.4 & 0.6974 \\
\hline Replicate Line [Sel. Reg. \& Inbr.] & 6,2394 & 37. & 7.0 & $<0.0001$ \\
\hline Adult Temperature & 1,2394 & 1177.7 & 21.8 & $<0.0001$ \\
\hline Adult Feeding Regime & 1,2394 & 52827.7 & 977.2 & $<0.0001$ \\
\hline Sex & 1,2394 & 3684.1 & 68.1 & $<0.0001$ \\
\hline Selection Reg. * Inbreeding Level & 2,6 & 475.6 & 1.3 & 0.3451 \\
\hline Selection Reg. * Adult Temp. & 1,2394 & 104.6 & 1.9 & 0.1644 \\
\hline Selection Reg. * Adult Feed. & 1,2394 & 49.3 & 0.9 & 0.3399 \\
\hline Selection Reg. * Sex & 1,2394 & 15.4 & 0.3 & 0.5936 \\
\hline Inbreeding Level * Adult Temp. & 2,2394 & 37.1 & 0.7 & 0.5033 \\
\hline Inbreeding Level * Adult Feed. & 2,2394 & 112.8 & 2.1 & 0.1244 \\
\hline
\end{tabular}

Table 4 Results of nested ANOVAs for longevity (Continued)

\begin{tabular}{lrrrc}
\hline Inbreeding Level * Sex & 2,2394 & 137.9 & 2.6 & 0.0782 \\
Adult Temp. * Adult Feed. & 1,2394 & 451.8 & 8.4 & $\mathbf{0 . 0 0 3 9}$ \\
Adult Temp. * Sex & 1,2394 & 114.8 & 2.1 & 0.1451 \\
Adult Feed. * Sex & 1,2394 & 1481.2 & 27.4 & $<0.0001$ \\
Sel. Reg. * Inbreed. * Adult Temp. & 2,2394 & 17.4 & 0.3 & 0.7248 \\
Sel. Reg. * Inbreed. *Adult Feed. & 2,2394 & 532.5 & 9.9 & $<0.0001$ \\
Sel. Reg. * Inbreed. * Sex & 22394 & 0.7 & $<0.1$ & 0.9879 \\
Sel. Reg. * Adult Temp. * Sex & 2,2394 & 1.7 & $<0.1$ & 0.8610 \\
Sel. Reg. * Adult Temp. * AdFeed. & 1,2394 & 128.8 & 2.4 & 0.1229 \\
Sel. Reg. * Adult Feed. * Sex & 1,2394 & 0.7 & $<0.1$ & 0.9110 \\
Inbreed. * Adult Temp. * AdFeed. & 2,2394 & 14.4 & 0.3 & 0.7659 \\
Inbreed. * Adult Temp. * Sex & 2,2394 & 161.9 & 3.0 & 0.0502 \\
Inbreed. * Adult Feed. * Sex & 2,2394 & 164.2 & 3.0 & $\mathbf{0 . 0 4 8 2}$ \\
Adult Temp. * Adult Feed. * Sex & 1,2394 & 87.1 & 1.6 & 0.2044 \\
Inbreed. * AdT * AdF * Sex & 2,2394 & 222.8 & 4.1 & $\mathbf{0 . 0 1 6 3}$ \\
Error & 2394 & 54.0 & & \\
\hline
\end{tabular}

Results for the effects of selection regime (selection vs. control), inbreeding level $(C, 11,12)$, replicate line, replicate cage, rearing temperature $\left(20 \mathrm{vs} .27^{\circ} \mathrm{C}\right)$, and sex on longevity in Bicyclus anynana (A). In Tables B (animals reared at $20^{\circ} \mathrm{C}$ ) and $C$ (animals reared at $27^{\circ} \mathrm{C}$ ) data were analyzed separately for each rearing temperature, as different feeding treatments were employed in animals reared at $27^{\circ} \mathrm{C}$ only, and as different adult temperatures were employed in animals reared at $27^{\circ} \mathrm{C}$ only temporarily. Consequently, the analysis in A was restricted to the animals fed ad libitum and kept as adults at $27^{\circ} \mathrm{C}$, to mainly explore effects of rearing temperature. The factor rearing temperature was replaced by the factor adult temperature in analyses B and $\mathrm{C}$, and the factor adult feeding regime (control vs. starvation) was added to Table C. For details see Material and Methods. Replicate line (random factor) was nested within selection regime and inbreeding level throughout. Minimum adequate models were constructed by sequentially removing non-significant interaction terms. Significant $P$-values are given in bold.

vs. $28.8 \pm 0.99 \mathrm{~min})$, and individuals acclimated to the lower temperature recovered faster than those acclimated to the higher temperature $(20.9 \pm 0.36 \mathrm{~min}<31.2$ $\pm 0.35 \mathrm{~min})$. The significant interactions found here basically reflect the patterns described above, and are thus not described here except for both interactions involving feeding regime. The response to adult feeding regime varied largely among inbreeding levels and sexes, with feeding regime having positive to negative effects (significant inbreeding level * adult feeding regime * sex interaction; Figure 2E). Furthermore, food stress had generally either little or positive effects on chill-coma recovery times in the adult temperature by sex groups, while it slightly decreased cold stress resistance in the females acclimated to $27^{\circ} \mathrm{C}$ (significant adult temperature " adult feeding regime * sex interaction; Figure 2F).

\section{Longevity}

In the analysis addressing effects of rearing temperature, longevity was significantly affected by rearing temperature and replicate line, but not by selection regime, inbreeding level, and sex (Table 4A). Animals 
reared at $20^{\circ} \mathrm{C}$ lived longer compared to animals reared at $27^{\circ} \mathrm{C}(29.9 \pm 0.33 \mathrm{~d}>12.3 \pm 0.25 \mathrm{~d})$. The significant selection regime by inbreeding level interaction indicates that longevity tended to be longer in the unselected controls than in the selection lines in the outbred controls $(23.2 \pm 0.78 \mathrm{~d}$ vs. $19.7 \pm 0.81 \mathrm{~d})$, but shorter in inbreeding group $1(20.5 \pm 0.81 \mathrm{~d}$ vs. $22.0 \pm 0.79 \mathrm{~d})$, while being very similar in inbreeding group $2(20.6 \pm 0.84 \mathrm{~d}$ vs. $20.9 \pm 0.87 \mathrm{~d}$ ). These patterns, however, were largely restricted to animals reared at $20^{\circ} \mathrm{C}$ and to males (significant selection regime * inbreeding level * rearing temperature interaction and significant selection regime * inbreeding level " sex interaction; Figure 3A and 3B). Consequently, significant differences between selection and control lines were exclusively found in outbred males reared at $20^{\circ} \mathrm{C}(35.5 \pm 1.1 \mathrm{~d}$ vs. $26.3 \pm 1.1 \mathrm{~d}$; significant four-way interaction). When being reared at $20^{\circ} \mathrm{C}$, longevity was longest in inbreeding 1 group (C: $30.0 \pm 0.56$; I1: $30.7 \pm 0.56 \mathrm{~d}$; I2: $29.0 \pm 0.61 \mathrm{~d}$ ), while it was shortest in this group when being reared at $27^{\circ} \mathrm{C}$ (C: $12.8 \pm 0.42$; I1: $11.7 \pm 0.42$ d; I2; $12.4 \pm 0.43$ d; significant inbreeding level * rearing temperature interaction). Sex differences were only found in animals reared at $20^{\circ} \mathrm{C}$ (males: $31.1 \pm 0.47 \mathrm{~d}>$ females: $28.8 \pm 0.47 \mathrm{~d}$ ), while there was no significant sex difference in animals reared at $27^{\circ} \mathrm{C}$ (males: $11.0 \pm 0.35 \mathrm{~d}=$ females: $13.7 \pm$ $0.35 \mathrm{~d}$; Tukey HSD; significant rearing temperature * sex interaction).

When considering the animals reared at $20^{\circ} \mathrm{C}$ separately, adult temperature, sex, and replicate line significantly affected longevity, but not selection regime and inbreeding level (Table 4B). Adults acclimated to $20^{\circ} \mathrm{C}$ lived longer than adults acclimated to $27^{\circ} \mathrm{C}(34.7 \pm 0.57$ $\mathrm{d}>24.4 \pm 0.62 \mathrm{~d}$ ), and males lived longer than females $(30.9 \pm 0.60 \mathrm{~d}>28.1 \pm 0.60 \mathrm{~d})$. The sex difference was restricted to the adult temperature of $27^{\circ} \mathrm{C}$ though (males: $27.3 \pm 0.87 \mathrm{~d}>$ females: $21.5 \pm 0.88 \mathrm{~d}$ ), while there was no significant difference at $20^{\circ} \mathrm{C}$ (males: $34.6 \pm$ $0.82 \mathrm{~d}=$ females: $34.7 \pm 0.80 \mathrm{~d}$; Tukey HSD; significant adult temperature * sex interaction). The significant interaction between selection regime and adult temperature indicates that longevity was longer in unselected controls compared to selection lines at the lower adult temperature $(36.2 \pm 0.80 \mathrm{~d}$ vs. $33.1 \pm 0.82 \mathrm{~d})$, but vice versa at the higher adult temperature $(23.8 \pm 0.86 \mathrm{~d}$ vs. $24.9 \pm 0.88 \mathrm{~d}$ ). Longevity decreased with increasing inbreeding level when animals were acclimated to $20^{\circ} \mathrm{C}$ (C: $37.2 \pm 0.98 \mathrm{~d}=\mathrm{I} 1: 35.8 \pm 0.94 \mathrm{~d}>\mathrm{I} 2: 31.0 \pm 1.05 \mathrm{~d}$ ), but tended to increase with increasing inbreeding level when animals were acclimated to $27^{\circ} \mathrm{C}(\mathrm{C}: 22.2 \pm 1.0 \mathrm{~d}=$ I1: $24.4 \pm 1.06 \mathrm{~d}=\mathrm{I} 2: 26.6 \pm 1.13 \mathrm{~d}$; Tukey HSD; significant inbreeding level * adult temperature interaction). Finally, males of the outbred control and the inbreeding 1 group showed quite substantial though opposing differences between selection lines and unselected controls, while differences where much smaller in other groups (significant selection regime * inbreeding level * sex interaction; Figure 3C).

In the animals reared at $27^{\circ} \mathrm{C}$, longevity was significantly affected by adult temperature, adult feeding regime, sex and replicate line, but not by selection regime and inbreeding level (Table 4C). Adults acclimated to
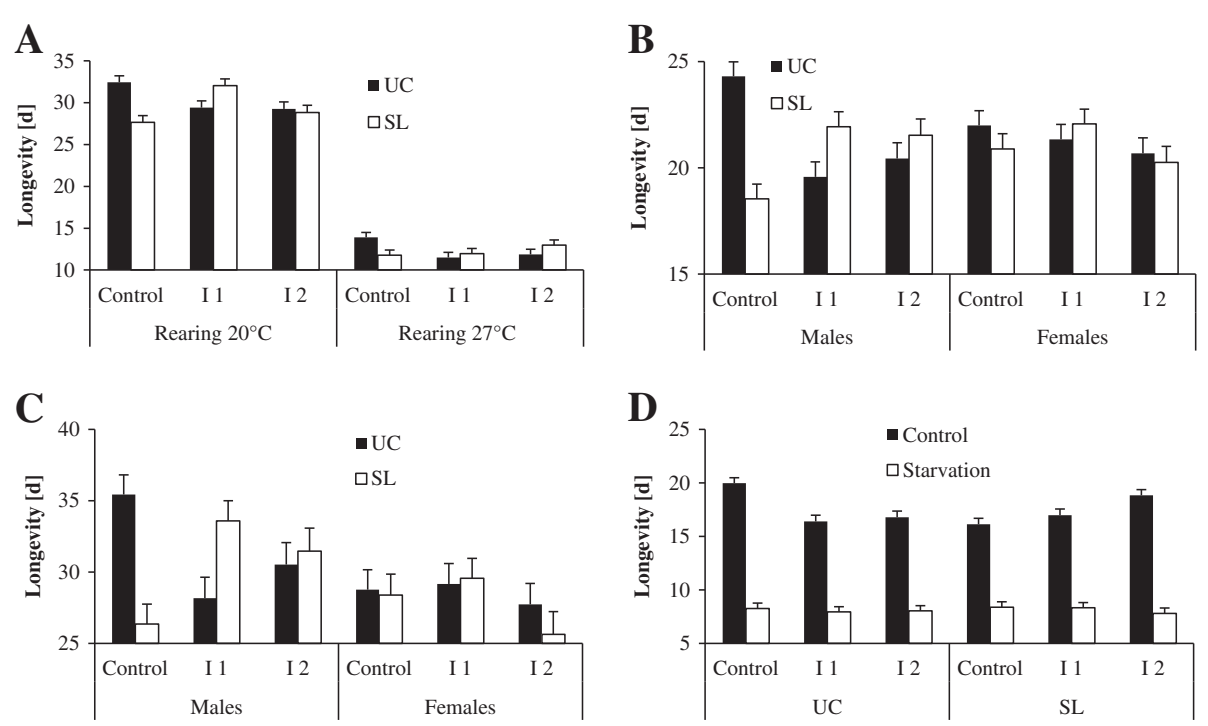

Figure 3 Variation in longevity (means $+1 \mathrm{SE}$ ) in Bicyclus anynana. Longevity in relation to rearing temperature, inbreeding level and selection regime $(\mathbf{A})$, sex, inbreeding level and selection regime $(\mathbf{B})$, sex, inbreeding level and selection regime $(\mathbf{C})$, and selection regime, inbreeding level and adult feeding treatment (D). Graphs A-B refer to animals that were fed ad libitum and kept at $27^{\circ} \mathrm{C}$ as adults, graph $\mathbf{C}$ to the animals reared at $20^{\circ} \mathrm{C}$, and graph $\mathrm{D}$ to those reared at $27^{\circ} \mathrm{C}$. UC: unselected control; SL: selection line. 
$20^{\circ} \mathrm{C}$ lived longer than adults acclimated to $27^{\circ} \mathrm{C}(13.5 \pm$ $0.21 \mathrm{~d}>12.1 \pm 0.21 \mathrm{~d}$ ), control animals lived longer compared to food-stressed animals $(17.5 \pm 0.22 \mathrm{~d}>8.1$ $\pm 0.20 \mathrm{~d})$, and females lived longer than males (14.1 \pm $0.21 \mathrm{~d}>11.6 \pm 0.21 \mathrm{~d})$. Effects of adult temperature were more pronounced in control $\left(20^{\circ} \mathrm{C}: 18.7 \pm 0.32 \mathrm{~d}\right.$ vs. $27^{\circ} \mathrm{C}$ : $16.4 \pm 0.31 \mathrm{~d})$ than in food-stressed animals $\left(20^{\circ} \mathrm{C}\right.$ : $8.4 \pm 0.29 \mathrm{~d}$ vs. $27^{\circ} \mathrm{C}: 7.8 \pm 0.28 \mathrm{~d}$; significant adult temperature * adult feeding regime interaction). Sex differences, in contrast, were larger in food-stressed (female: $10.2 \pm 0.29 \mathrm{~d}$ vs. male: $6.1 \pm 0.28 \mathrm{~d}$ ) than in control animals (female: $18.0 \pm 0.32 \mathrm{~d}$ vs. male: $17.1 \pm$ $0.31 \mathrm{~d}$; significant adult feeding regime * sex interaction). While in food-stressed animals longevity was generally very similar across selection regimes and inbreeding levels, the feeding control groups showed divergent patterns between unselected controls and selection lines (Figure 3D). While in the latter groups longevity increased with increasing inbreeding level in the selection lines $(\mathrm{C}: 16.1 \pm 0.56 \mathrm{~d}=\mathrm{I} 1: 16.9 \pm 0.56 \mathrm{~d}<\mathrm{I} 2$ : $18.8 \pm 0.54 \mathrm{~d}$ ), it decreased with increasing inbreeding level in the unselected control lines (C: $20.2 \pm 0.51 \mathrm{~d}>\mathrm{I} 1$ : $16.4 \pm 0.56 \mathrm{~d}=\mathrm{I} 2: 16.8 \pm 0.56 \mathrm{~d}$; significant selection regime * inbreeding level * adult feeding regime interaction). The significant inbreeding level " adult feeding regime * sex interaction shows that inbreeding effects were only present in food control males, in which individuals from inbreeding level 1 lived shortest (I1: $15.5 \pm$ $0.56 \mathrm{~d}<\mathrm{I} 2: 17.0 \pm 0.55 \mathrm{~d}=\mathrm{C}: 18.7 \pm 0.53 \mathrm{~d})$. Variation between inbreeding groups though was restricted to food control males kept at $20^{\circ} \mathrm{C}$ (I1: $16.1 \pm 0.79$ d; I2: $18.1 \pm$ $0.78 \mathrm{~d}$; C: $21.6 \pm 0.76 \mathrm{~d}$; significant inbreeding level * adult temperature " adult feeding regime " sex interaction).

\section{Discussion}

\section{Variation in developmental traits}

As predicted (Table 1), selection regime neither affected larval time, pupal time, larval growth rate or pupal mass, thus suggesting that genetic variation in adult cold tolerance operates largely independent of these developmental traits in B. anynana (see [17]; cf. [16,42]). However, selection regime was involved in a total of six interactions with other factors (larval time: 3; pupal time: 1; growth rate: 1; pupal mass: 1; Table 2). The patterns revealed through these interactions though do not fundamentally change the conclusion drawn above, with perhaps the following exception. Larval development time was slightly longer in control than in selection lines at the lower rearing temperature only. This may indicate that the lines selected for increased adult cold tolerance may be slightly better adapted to deal with developmental temperatures below the optimal range. Note though that this pattern was not consistent across all treatment groups (significant 3-way interaction). All other interactions indicated inconsistent, group-specific responses and did therefore not reveal any straightforward effects of selection regime on developmental traits.

Likewise, inbreeding yielded only marginal effects, significantly affecting pupal mass only. Pupal mass was highest in inbreeding group 1, intermediate in the outbred control, and lowest in inbreeding group 2. Directly after the full-sib matings, in contrast, larval development time, larval growth rate, and pupal mass showed inbreeding depression [17]. The fact that much of these initial differences disappeared indicates fitness rebounds caused by subsequent random mating (for 14 generations: 10 during the selection experiments and 4 afterwards) as was expected [17]. Additive theory would predict a complete rebound under such circumstances $[43,44]$, such that the pattern found for pupal mass may reflect a chance effect caused by random genetic drift rather than inbreeding depression. Note in this context that the 11 interactions in which inbreeding was involved in also indicated erratic variation rather than any systematic patterns.

In contrast to the above results, sexual differences and variation induced by different rearing temperatures resemble predicted and well-known patterns for B. anynana and beyond $[45,46]$. Specifically, lower temperatures resulted in increased development time and pupal mass, but in decreased larval growth rates. This is consistent with the temperature-size rule and the in general temperaturedependent growth of ectotherms [46-48]. Males compared to females showed shorter larval but longer pupal times (though with the latter not affecting earlier male emergence; protandry selection), higher larval growth rate, and lower pupal mass (fecundity selection in females). Sex differences were more pronounced at $20^{\circ} \mathrm{C}$ (larval time) or at $27^{\circ} \mathrm{C}$ (pupal time, larval growth rates). While the former has been reported previously and likely reflects larger absolute differences owing to the overall longer development time at lower temperatures [49], we have no explanation for the latter.

\section{Variation in chill-coma recovery time}

Cold tolerance was strongly affected by selection regime, with recovery times being overall by $21 \%$ shorter in the selection than in the control lines. Directly after the course of selection the difference between selection regimes was 29\% [17], indicating an ongoing convergence towards control line levels. This suggests that the selected genes and alleles had not yet become fixed in the selection lines, and furthermore a lack of selection for maintaining increased cold tolerance under laboratory conditions. Interestingly, the differences between selection and control lines tended to be more pronounced when animals had been reared at $20^{\circ} \mathrm{C}(23 \%)$ as 
compared to $27^{\circ} \mathrm{C}$ (18\%; Table $\left.3 \mathrm{~A}\right)$. This suggests that the selection lines might show a somewhat stronger response to rearing temperature (by $43 \%$ shorter chillcoma recovery time at $20^{\circ} \mathrm{C}$ compared to $27^{\circ} \mathrm{C}$ ) than the control lines (by $40 \%$ shorter).

Regarding interactive effects, selection regime was involved in a total of five significant interactions. While three of these did not reveal any conclusive patterns regarding the role of selection regime, the remaining two interactions suggest that the differences between selection and control lines were larger at the higher as compared to the lower adult temperature (Table $3 \mathrm{~A}$ and B). However, while this is true in absolute terms (5.8 minutes at $27^{\circ} \mathrm{C}$ vs. 3.7 minutes at $20^{\circ} \mathrm{C}$ for all individuals; 5.9 minutes at $27^{\circ} \mathrm{C}$ vs. 2.1 minutes at $20^{\circ} \mathrm{C}$ for the individuals reared at $20^{\circ} \mathrm{C}$ ), larger relative differences were found in the animals reared at $20^{\circ} \mathrm{C}$ only $\left(27^{\circ} \mathrm{C}\right.$ : $20 \%$ and $20^{\circ} \mathrm{C}: 21 \%$ for all; $27^{\circ} \mathrm{C}$ : $25 \%$ and $20^{\circ} \mathrm{C}$ : $19 \%$ for individuals reared at $20^{\circ} \mathrm{C}$ ). Concomitantly plastic responses are quite similar across selection regimes, with the selection lines showing by $41 \%$ (all) and $48 \%$ (reared at $20^{\circ} \mathrm{C}$ ) shorter chill-coma recovery times at $20^{\circ} \mathrm{C} \mathrm{com-}$ pared to $27^{\circ} \mathrm{C}$, and the control lines by $40 \%$ and $52 \%$ shorter ones. We therefore conclude that the extent of the plastic responses in cold tolerance is comparably high across both selection regimes, thus challenging the main hypothesis we wanted to test here.

Effects of different rearing and adult temperatures were strong and persistent, with animals reared or acclimated at the lower temperature showing increased cold tolerance compared to those kept at the higher temperature (cf. Table 1). Similar temperature-induced changes in cold tolerance have been documented in a large variety of insects, suggesting a universal pattern of adaptive phenotypic plasticity $[23,50]$. In contrast, no consistent effects of adult feeding treatment on cold tolerance were found, despite large variation among inbreeding levels, sexes, and temperature groups. This is in line with our predictions based on earlier results [23], which indicates that cold tolerance is at least not strongly limited by adult food intake. Furthermore, our results revealed no sex differences in cold tolerance as was expected. Likewise, testing for sex differences in temperature stress resistance in an earlier study yielded a non-significant result in 18 out of 22 statistical analyses, suggesting that, in general, both sexes are equally tolerant in B. anynana [23]. Except from some groupspecific responses, no effects of (earlier) inbreeding on cold tolerance were found.

\section{Variation in longevity}

Longevity was not affected by selection regime, indicating that there is no trade-off between increased cold stress resistance and life span in B. anynana (cf. [34]).
This notion is further supported by the erratic patterns indicated by the seven interactions in which selection regime was involved in. Studies on Drosophila also yielded inconclusive results regarding genetic correlations between cold tolerance and longevity (e.g. [2,15,16,42]). Temperature effects, in contrast, were straight-forward and as predicted. Animals reared at or acclimated to $20^{\circ} \mathrm{C}$ lived generally longer than animals reared or kept at the higher temperature, thus indicating accelerated rates of ageing at warmer temperatures owing to higher metabolic rates $[2,47,51]$. Adult food stress had as expected a large impact on longevity, with a lack of food substantially reducing life span $[36,37]$. Not surprisingly, differences in longevity between acclimation temperature groups were more pronounced in control compared to food-stressed animals, as all latter individuals died within relatively short time spans. Once again, inbreeding yielded no conclusive effects.

Regarding sex differences, females lived longer than males when being reared at $27^{\circ} \mathrm{C}$, as has been found in several earlier studies [17,38]. The sex difference was more pronounced in food-stressed than in control animals, which may suggest a re-allocation of resources from reproduction to somatic maintenance under food stress in females. When being reared at $20^{\circ} \mathrm{C}$, however, males lived longer than females, but only at the higher adult temperature. This finding may suggest that the respective temperature regime does not only affect longevity as such, but influences sexes in different ways.

\section{Conclusions}

Regarding effects of temperature, feeding treatment, sex, inbreeding and selection regime our results are in good agreement with the hypotheses outlined in Table 1 and thus with earlier findings obtained in B. anynana and other insects. However, our principal intention here was to explore plastic responses in populations of different genetic background and thus genotype-environment interactions. Regarding this matter we have indeed detected a number of interactions in which selection regime was involved in, but most of these did not reveal consistent patterns. We have found some evidence though that the lines selected for increased cold tolerance as compared to control lines (1) developed slightly faster at suboptimally low temperatures, (2) showed larger differences in cold tolerance compared to controls when being reared at the lower temperature, (3) and showed more pronounced differences in cold tolerance compared to controls at an adult temperature of $27^{\circ} \mathrm{C}$. The former findings may indicate that the lines selected for increased adult cold tolerance may be slightly better equipped to deal with lower temperatures, such that any advantages become exaggerated when experiencing suboptimally low temperatures during development. While 
thus the plastic response to different rearing temperatures seemed to be somewhat larger in the selection compared to the control lines, plastic responses to different adult temperatures were very similar across selection regimes. Our results thus suggest that plastic capacities were, if anything, only marginally affected by directional selection, and that plasticity was largely independent of the respective trait mean. This suggests that the genetic architecture of the trait mean operates largely independently of the architecture underlying plastic responses. Thus, we found no evidence for an interference of genetic adaptation with plastic capacities (but see [52] for an interspecific comparison).

However, one should keep in mind that our study draws on variation across selection lines, which had been established from a single laboratory source population. While using a single gene pool to experimentally establish genetically differentiated populations has clear advantages, it also involves some limitations which should be acknowledged. Notably, we do not know whether our results are transferable to other populations (and species), and whether they might be affected by the specific population history (e.g. inbreeding, founder effects, laboratory adaptation). Several traits have been shown to readily respond to laboratory conditions, though essentially nothing is known about the effects of laboratory adaptation on plastic responses [53,54]. For logistic reasons such limitations apply to many laboratory experiments. At least some studies indicate that laboratory results may nevertheless have clear relevance for field conditions [54]. In any case our results clearly show that there is not necessarily a link between trait value and the magnitude of plastic responses.

\section{Competing interests}

The authors declare that they have no competing interests.

\section{Authors' contributions}

$\mathrm{KFr}$ conducted the experiment, performed the statistical analyses and drafted the manuscript. AD performed the selection experiment. KFi conceived the study, performed the statistical analyses and helped to draft the manuscript. All authors read and approved the final manuscript.

\section{Acknowledgements}

We thank two anonymous reviewers for helpful suggestions and C. Michalowsky, H. Höltje, V. Herkules, M. Piesk, S. Bauerfeind, and T. Kehl for helping during the experiment. This work was supported by the University of Greifswald and the Landesgraduiertenförderung of Mecklenburg Western Pomerania.

Received: 4 July 2012 Accepted: 27 November 2012

Published: 5 December 2012

\section{References}

1. David JR, Gibert P, Pla E, Petavy G, Karan D, Moreteau B: Cold stress tolerance in Drosophila: Analysis of chill coma recovery in D. melanogaster. J Therm Biol 1998, 23:291-299.

2. Anderson AR, Hoffmann AA, Mckechnie SW: Response to selection for rapid chill-coma recovery in Drosophila melanogaster: physiology and life-history traits. Genet Res 2005, 85:5-22.
3. Hoffmann AA, Sorensen JG, Loeschcke V: Adaptation of Drosophila to temperature extremes: bringing together quantitative and molecular approaches. J Therm Biol 2003, 28:175-216.

4. Ayrinhac A, Debat V, Gibert P, Kister AG, Legout H, Moreteau B, Vergilino R, David JR: Cold adaptation in geographical populations of Drosophila melanogaster: phenotypic plasticity is more important than genetic variability. Funct Ecol 2004, 18:700-706.

5. Fischer K, Karl I: Exploring plastic and genetic responses to temperature variation using copper butterflies. Clim Res 2010, 43:17-30.

6. David JR, Gibert P, Moreteau B, Gilchrist GW, Huey RB: The fly that came in from the cold: geographic variation of recovery time from low-temperature exposure in Drosophila subobscura. Funct Ecol 2003, 17:425-430.

7. Castañeda LE, Lardies MA, Bozinovic F: Interpopulational variation in recovery time from chill coma along a geographic gradient: A study in the common woodlouse, Porcellio laevis. J Insect Physiol 2005, 51:1346-1351

8. Pigliucci M: Phenotypic Plasticity: Beyond Nature and Nurture. Baltimore, MD: Johns Hopkins University Press; 2001.

9. Hoffmann AA, Anderson A, Hallas R: Opposing clines for high and low temperature resistance in Drosophila melanogaster. Ecol Lett 2002, 5:614-618.

10. Hazell SP, Groutides C, Neve BP, Blackburn TM, Bale JS: A comparison of low temperature tolerance traits between closely related aphids from the tropics, temperate zone, and Arctic. J Insect Physiol 2010, 56:115-122

11. Klok CJ, Chown SL: Resistance to temperature extremes in sub-Antarctic weevils: interspecific variation, population differentiation and acclimation. Bio J Linn Soc 2003, 78:401-414.

12. Hazell SP, Neve BP, Groutides C, Douglas AE, Blackburn TM, Bale JS: Hyperthermic aphids: Insights into behaviour and mortality. J Insect Physiol 2010, 56:123-131.

13. Chown SL, Klok CJ: Altitudinal body size clines: latitudinal effects associated with changing seasonality. Ecography 2003, 26:445-455.

14. Karl I, Janowitz SA, Fischer K: Altitudinal life-history variation and thermal adaptation in the copper butterfly Lycaena tityrus. Oikos 2008, 117:778-788.

15. Bubliy OA, Loeschcke $\mathrm{V}$ : Correlated responses to selection for stress resistance and longevity in a laboratory population of Drosophila melanogaster. J Evol Biol 2005, 18:789-803.

16. Bertoli Cl, Scannapieco AC, Sambucetti P, Norry FM: Direct and correlated responses to chill-coma recovery selection in Drosophila buzzatii. Entomol Exp Appl 2010, 134:154-159.

17. Dierks A, Hoffmann B, Bauerfeind SS, Fischer K: Effects of inbreeding on life history and thermal performance in the tropical butterfly Bicyclus anynana. Popul Ecol 2012, 54:83-90

18. Chidawanyika F, Terblanche JS: Rapid thermal responses and thermal tolerance in adult codling moth Cydia pomonella (Lepidoptera: Tortricidae). J Insect Physiol 2011, 57:108-117.

19. Chidawanyika F, Terblanche JS: Costs and benefits of thermal acclimation for codling moth, Cydia pomonella (Lepidoptera: Tortricidae): implications for pest control and the sterile insect release programme. Evol Appl 2011, 4:534-544.

20. Fischer K, Eenhoorn E, Bot ANM, Brakefield PM, Zwaan BJ: Cooler butterflies lay larger eggs: developmental plasticity versus acclimation. Proc $R$ SoC Lond B-Bio 2003, 270:2051-2056.

21. Lee $R E$, Chen $C P$, Denlinger $D L$ : A rapid cold-hardening process in insects. Science 1987, 238:1415-1417.

22. Basson CH, Nyamukondiwa C, Terblanche JS: Fitness costs of rapid coldhardening in Ceratitis capitata. Evolution 2012, 66:296-304.

23. Fischer K, Dierks A, Franke K, Geister TL, Liszka M, Winter S, Pflicke C: Environmental effects on temperature stress resistance in the tropical butterfly Bicyclus anynana. Plos One 2010, 5(12):e15284.

24. Brian PL, Heather AF, James GL Christopher PY: Genotype-by-environment interactions and adaptation to local temperature affect immunity and fecundity in Drosophila melanogaster. Plos Pathog, 4(3):1000025.

25. Scheiner SM, Lyman RF: The genetics of phenotypic plasticity. II. Response to selection. J Evol Biol 1991, 4:23-50.

26. Stearns SC: The Evolution of life histories. Oxford: University Press; 1992.

27. Scheiner SM: Genetics and evolution of phenotypic plasticity. Annu Rev Ecol Syst 1993, 24:35-68. 
28. Falconer DS, Mackay TFC: Introduction to quantitative genetics. Essex: Longman; 1996.

29. Brakefield PM, Kesbeke F: Genotype-environment interactions for insect growth in constant and fluctuating temperature regimes. Proc $R$ Soc Lond B-Bio 1997, 264:717-723.

30. Danielson-Francois AM, Kelly JK, Greenfield MD: Genotype x environment interaction for male attractiveness in an acoustic moth: Evidence for plasticity and canalization. J Evol Biol 2006, 19:532-542.

31. Fanara JJ, Folguera G, Iriarte PF, Mensch J, Hasson E: Genotype by environment interactions in viability and developmental time in populations of cactophilic Drosophila. J Evol Biol 2006, 19:900-908.

32. Gibert P, Moreteau B, Petavy G, Karan D, David JR: Chill-coma tolerance, a major climatic adaptation among Drosophila species. Evolution 2001 55:1063-1068.

33. Terblanche JS, Hoffmann AA, Mitchell KA, Rako L, le Roux PC, Chown SL: Ecologically relevant measures of tolerance to potentially lethal temperatures. J Exp Biol 2011, 214:3713-3725.

34. Dierks A, Baumann B, Fischer K: Response to selection on cold tolerance is constrained by inbreeding. Evolution 2012, 66:2384-2398.

35. Dierks A, Kölzow N, Franke K, Fischer K: Does selection on increased cold tolerance in the adult stage confer resistance throughout development? $J$ Evol Biol, . in press.

36. Bauerfeind SS, Fischer K: Effects of food stress and density in different life stages on reproduction in a butterfly. Oikos 2005, 111:514-524.

37. Bauerfeind SS, Perlick JEC, Fischer K: Disentangling environmental effects on adult life span in a butterfly across the metamorphic boundary. Exp Gerontol 2009, 44:805-811.

38. Larsen TB: The butterflies of Kenya. Oxford: Oxford University Press; 1991.

39. Lyytinen A, Brakefield PM, Lindstrom L, Mappes J: Does prediction maintain eyespot plasticity in Bicyclus anynana? Proc $R$ Soc Lond B-Bio 2004, 271:279-283.

40. Brakefield PM, El Filali E, Van der Laan R, Breuker CJ, Saccheri IJ, Zwaan B: Effective population size, reproductive success and sperm precedence in: the butterfly, Bicyclus anynana, in captivity. J Evol Biol 2001, 14:148-156.

41. Van't Hof AE, Zwaan BJ, Saccheri IJ, Daly D, Bot ANM, Brakefield PM: Characterization of 28 microsatellite loci for the butterfly Bicyclus anynana. Mol Ecol Notes 2005, 5:169-172.

42. Mori N, Kimura MT: Selection for rapid and slow chill and heat coma in Drosophila melanogaster. Biol J Linn Soc 2008, 95:72-80.

43. Wade MJ, Shuster SM, Stevens L: Inbreeding: Its effect on response to selection for pupal weight and the heritable variance in fitness in the flour beetle, Tribolium castaneum. Evolution 1996, 50:723-733.

44. Bouzat JL: Conservation genetics of population bottlenecks: the role of chance, selection, and history. Conserv Genet 2010, 11:463-478.

45. Fischer K, Fiedler K: Sex-related differences in reaction norms in the butterfly Lycaena tityrus (Lepidoptera: Lycaenidae). Oikos 2000, 90:372-380

46. Angilletta MJ, Steury TD, Sears MW: Temperature, growth rate, and body size in ectotherms: Fitting pieces of a life-history puzzle. Integr Comp Biol 2004, 44:498-509.

47. Kingsolver JG, Huey RB: Size, temperature, and fitness: three rules. Evol Ecol Res 2008, 10:251-268.

48. Walters RJ, Hassall M: The temperature-size rule in ectotherms: May a general explanation exist after all? Am Nat 2006, 167:775-775.

49. Karl I, Fischer K: Why get big in the cold? Towards a solution to a life-history puzzle. Oecologia 2008, 155:215-225.

50. Sejerkilde M, Sørensen JG, Loeschcke V: Effects of cold- and heat hardening on thermal resistance in Drosophila melanogaster. J Insect Physiol 2003, 49:719-726.

51. Kristensen TN, Hoffmann AA, Overgaard J, Sørensen JG, Hallas R, Loeschcke $\mathrm{V}$ : Costs and benefits of cold acclimation in field-released Drosophila. Proc Natl Acad Sci USA 2008, 105:216-221.

52. Nyamukondiwa C, Terblanche JS, Marshall KE, Sinclair BJ: Basal cold but not heat tolerance constrains plasticity among Drosophila species (Diptera: Drosophilidae). J Evol Biol 2011, 24:1927-1928.
53. Simões $P$, Rose MR, Duarte A, Gonçalves R, Matos M: Evolutionary domestication in Drosophila subobscura. J Evol Biol 2007, 20:758-766

54. Simões P, Santos J, Fragata I, Mueller LD, Rose MR, Matos M: How repeatable is adaptive evolution? The role of geographical origin and founder effects in laboratory adaptation. Evolution 2008, 62:1817-1829.

doi:10.1186/1471-2148-12-235

Cite this article as: Franke et al:: Directional selection on cold tolerance does not constrain plastic capacity in a butterfly. BMC Evolutionary Biology 2012 12:235.

\section{Submit your next manuscript to BioMed Central and take full advantage of:}

- Convenient online submission

- Thorough peer review

- No space constraints or color figure charges

- Immediate publication on acceptance

- Inclusion in PubMed, CAS, Scopus and Google Scholar

- Research which is freely available for redistribution 